\title{
Rituximab for refractory SLE_-patients reach lasting remission with short-term regimen
}

"The rationale for our approach," says Dario Roccatello, "is based on the need to induce a rapid response in critically ill patients, all the while ensuring them long-term remission without any further immunosuppressive therapy." And this need, it seems, is one that Roccatello et al. might have met, according to the results of an uncontrolled study of rituximab combination therapy in eight patients with severe or refractory systemic lupus erythematosus (SLE), showing B-cell depletion in all eight patients within 5 weeks, followed by lasting remission.

\section{4 ...showing B-cell depletion in all eight patients within 5 weeks... 77}

The anti-CD20 antibody rituximab induces apoptosis of CD20 ${ }^{+}$cells -including most B-cell subsets and a small population of T cells-which are central to the pathogeneis of SLE. Existing immunosuppressive therapies for SLE can be toxic or ineffective, but, despite reports that rituximab ameliorates several manifestations of SLE, two randomized, controlled trials (RCTs) of combination therapy in patients with moderate-tosevere SLE "failed to demonstrate any statistically significant differences between rituximab and placebo with regard to efficacy", says Roccatello. So why persist with testing the drug in patients with SLE? Patients refractory or intolerant to standard immunosuppressive drugs (and most in need of alternative therapy) Roccatello explains, are "relatively often seen in clinical practice", but are unlikely to be enrolled in RCTs.

The investigators administered rituximab intravenously at a dose of $375 \mathrm{mg} / \mathrm{m}^{2}$ on days 2, 8, 15 and 22, and again 1 month and 2 months later. The biologic agent was accompanied by two intravenous pulses of $750 \mathrm{mg}$ cyclophosphamide on days 4 and 7 and three pulses of methylprednisone $15 \mathrm{mg} / \mathrm{kg}$ on days 1,4 and 8 , followed by oral prednisone $50 \mathrm{mg}$ for 2 weeks, rapidly tapered to $5 \mathrm{mg}$. "Our own experience with other immune-mediated diseases showed that this scheme was associated with a delayed occurrence of relapses", says Roccatello, adding that its strength lies in the brevity of immunosuppression.

With a mean follow-up of 36.2 months, two of the eight patients experienced relapse, but retreatment with the same regimen was successful. One of these patients was the only one to develop anti-rituximab antibodies, and no serious adverse events occurred.

Emma Leah

Original article Roccatello, D. et al. Intensive short-term treatment with rituximab, cyclophosphamide and methylprednisolone pulses induces remission in severe cases of SLE with nephritis and avoids further immunosuppressive maintenance therapy. Nephrol. Dial. Transplant. doi:10.1093/ndt/gfr109 